\title{
37. AfnP-Symposium 2015 in Fulda Ein Rückblick auf die Veranstaltung
}

Vom 27.-28.11.2015 fand das Symposium der AfnP e.V. traditionell im MARITIM Hotel in Fulda statt. Es standen 2 volle Tage für interessante Vorträge zur Verfügung. Das Symposium wurde sehr gut besucht.

Erstmalig nutzten 148 Teilnehmer die Möglichkeit, sich im Vorfeld anzumelden und die Tagungsgebühr zu überweisen. Das entspannt die Situation an der Tageskasse. Kurzfristig kamen noch 64 Teilnehmer unangemeldet. Die Teilnehmer konnten an den 2 Veranstaltungstagen zwischen den in 3 Tagungsräumen parallel laufenden Vorträgen und Workshops aus den verschiedensten Themen auswählen.

\section{Breites Themenspektrum}

Das Programm sprach alle Berufsgruppen, erfahrene Pflegekräfte oder "Neulinge“ (Ärzte, Techniker, MFA etc.), die in der Nephrologie arbeiten, an. Die Vorträge waren insgesamt sehr gut besucht und von hoher Qualität. Die Organisatoren des Symposiums versuchten hierbei, immer alle Berufsgruppen und pflegerischen Bereiche in der Nephrologie anzusprechen. Das
Spektrum reichte deshalb von medizinischen Themen über pflegerische, ethische, berufspolitische, rechtliche bis hin $\mathrm{zu}$ technisch-praktischen Themen.

\section{Favoritenliste}

Die Favoritenliste an Symposiumsbeiträgen liest sich wie folgt:

- „Der Säure-Basen-Haushalt“, gehalten von Prof. Joachim Hoyer, Marburg, war der „absolute Hit“.

- „Medikamente der Dialysepatienten“, referiert von Prof. Frieder Keller, Ulm, der kurzfristig für die geplante Referentin eingesprungen ist, war eine Bereicherung.

- „Die Wunduhr“ von Gunar Riepe, Koblenz, wird künftig wieder als längerer Workshop angeboten.

- „Die wunderbare Welt der Doppelpumpen und alles zur Single-Needle“ gab so viele Denkanstöße und Fragen, dass wir diesen Punkt auch wiederholen werden.

- „Transplantation“ von Dr. Miriam Opgenoorth, Dresden

- PD-Vorträge von Gaby Ausobsky, Düsseldorf, Marion Bundschu, Ulm, sowie den Firmen Baxter und Fresenius
- „Natriumprofile“ und „Intradialytische Hypotonie“, vorgetragen von den Erst- und Zweitplatzierten beim Förderpreis Nephrologische Pflege des Jahres 2014 Carmen Reißenweber, Spardorf, und Beate Schumacher, Heilbronn

- Vorträge zu den Themen „Gefäßzugange“, „Shuntchirugie“ und „Selbstpunktion mit Erfahrungsbericht einer langjährigen Heimhämodialysepatientin“

- „Haftungsrecht“: Hier wurde nach dem 60-minütigen Vortrag noch 45 min diskutiert, obwohl es der letzte Vortrag am Freitagnachmittag war.

- „Lipoproteinapherese“

- „Ernährung am Lebensende“

- "Naturheilkunde“

- „Erstellung eines Patientenleitfadens mit praktischer Umsetzung“ von Rebecca Fiedler, Fulda, und Antje Dietrich, Altenstadt: Beide haben mit dieser gemeinsamen Arbeit den Förderpreis Nephrologische Pflege 2013 gewonnen. Die beiden Referentinnen wurden anschließend belagert mit der Frage, wie man an diese Unterlagen kommen kann. Die AfnP e.V. wird den Leitfaden in gedruckter Form zur Verfügung stellen.

Diese Aufzählung zeigt, wie wichtig Themen mit engem Praxisbezug für die Teilnehmer sind. Sie diskutierten lebhaft und stellten viele Fragen zum Berufsalltag. Da aus Zeitmangel und wegen Personaleinsparungen nicht mehr genug Zeit für eine gute Einarbeitung zur Verfügung steht, versuchen wir, diese Lücke mit entsprechenden Vorträgen zu schließen.

Pflegefehler können sehr schnell die Patientensicherheit gefährden. Bedienungsfehler an den Geräten führen zunehmend zum Austausch von völlig intakten Geräten, hier besteht Schulungsbedarf. Mer-

Abb. 1 Zufriedenheit mit der Auswahl der Themen.

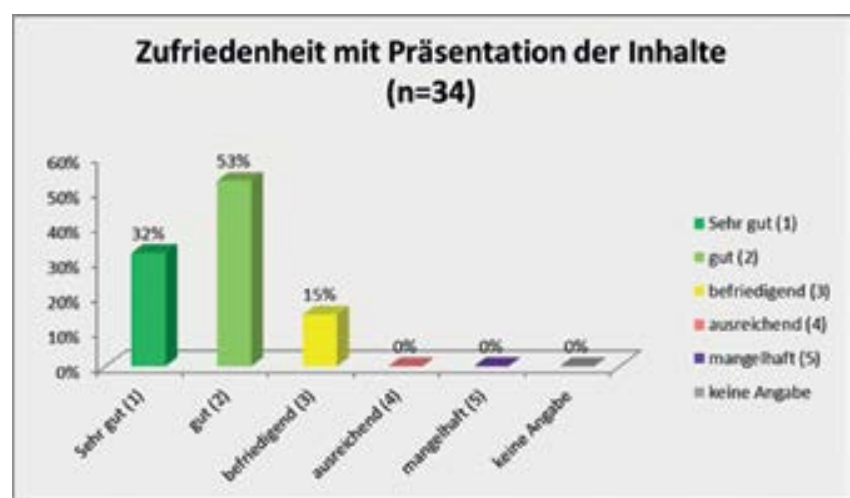

Abb. 2 Zufriedenheit mit der Präsentation der Inhalte.

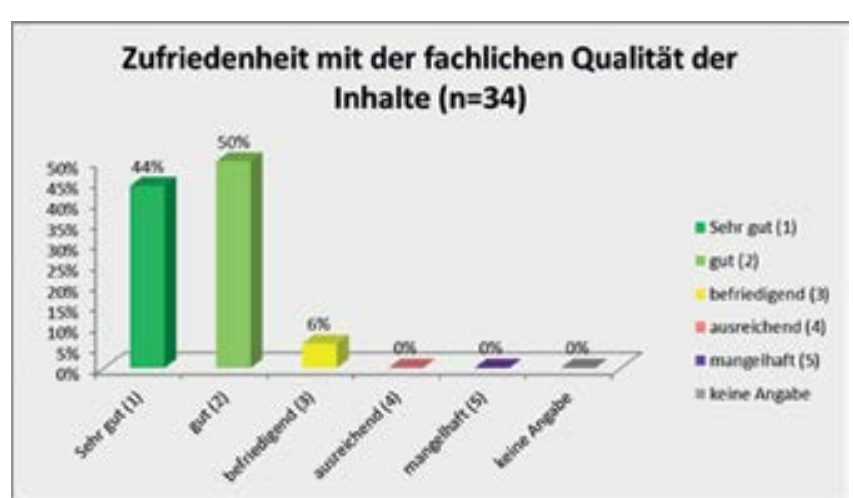

Abb. 3 Zufriedenheit mit der fachlichen Qualität der Inhalte. 
ken Sie sich schon jetzt den Termin der nächsten AfnP Dialyse Basic Days am 25. und 26.11.2016 vor, diesmal in Ulm.

\section{Teilnehmerbefragung}

Ein besonderer Dank geht an alle Teilnehmer, die uns ein ausführliches Feedback gegeben haben. Gerade die Liste mit Themenwünschen ist für uns sehr hilfreich und bereits in Bearbeitung. Einen Auszug aus der Befragung der Teilnehmer finden Sie in den Abbildungen 1-3. Die im „Forum Technik" angebotenen Themen wurden als besonders praxisnah gelobt. Selbst alte Hasen haben noch dazugelernt und Denkanstöße erhalten.

Der „Peritonealdialyse Basic Day“ wurde besonders gut angenommen, da er allen einen Überblick über die PD ermöglichte, die noch nicht in der PD arbeiten und somit keinen Zugang zu einer PD-Fortbildung haben. Die Verknüpfung der Theorie mit der Praxis vor Ort war besonders interessant. Dieser Tag wurde zu einem festen Programmpunkt beim Symposium.

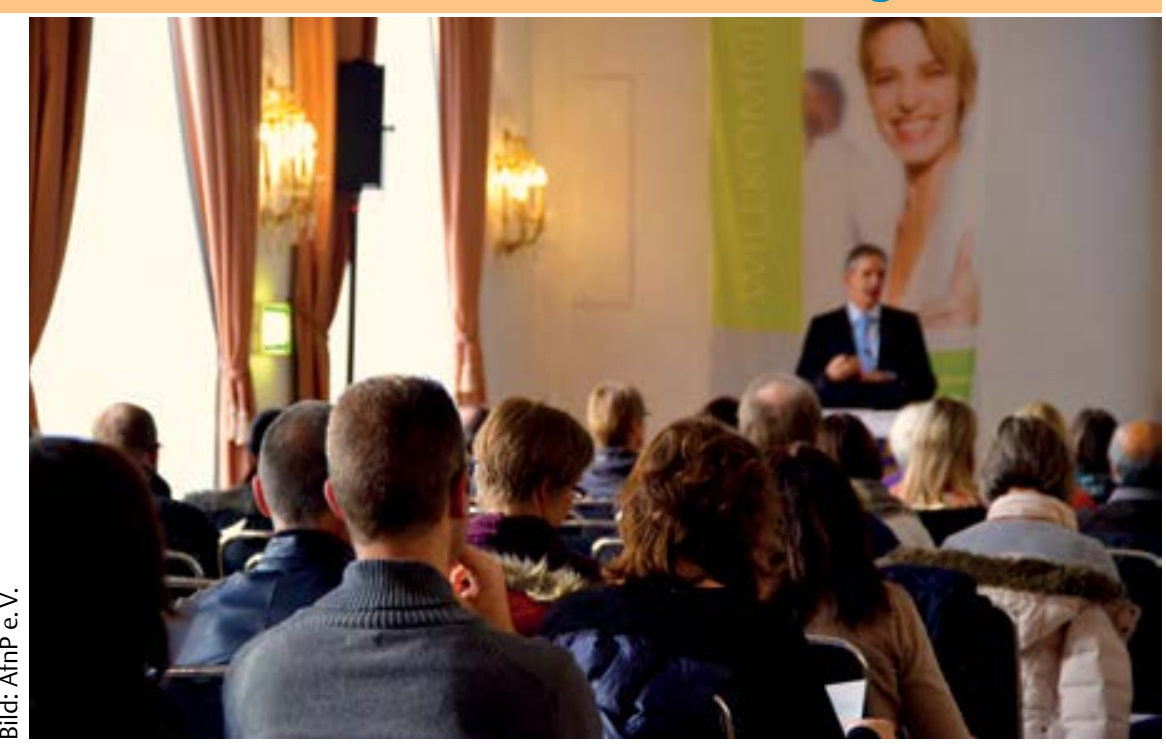

Einige Teilnehmer bemängelten den Tagungsort. Wir sehen seit einigen Jahren einen unverhältnismäßigen Anstieg der Kosten und werden daher den Tagungsort künftig verlegen. Unser Bestreben ist es, in der Mitte Deutschlands zu bleiben und wir sondieren gerade 3 sehr gute Angebote. Unser Augenmerk richtet sich auf be- zahlbare Übernachtungsmöglichkeiten und Verpflegung sowie eine gute Erreichbarkeit. Wir danken an dieser Stelle noch einmal allen fleißigen Helfern und Organisatoren, die durch ihr ehrenamtliches Engagement diese Veranstaltung wieder zu einem Erfolg geführt haben.

Marion Bundschu, Ulm 